\title{
Effets prévisibles en surface de galeries minières creusées à faible profondeur dans les grès vosgiens. Exemple du traitement de la galerie de Falck
}

\section{F. WOJTKOWIAK}

INERIS LAEGO

École nationale supérieure des mines

Parc de Saurupt

54042 Nancy Cedex

alain.thoraval@ineris.fr francis.wojtkowiak@ineris.fr

M. METZ

Houillères du Bassin de Lorraine, UGEPI

2, rue de Metz 57802 Freyming-Merlebach Cedex michel.metz@hbl.fr
Les conditions de stabilité des galeries creusées dans les grès vosgiens, modifiées à la suite d'une dégradation progressive de leur recouvrement et/ou de la mise en eau de ces galeries, ont été analysées. Les effets des instabilités potentielles induits éventuellement en surface du sol ont été également quantifiés. Dans le cas le plus défavorable, les analyses réalisées prédisent l'apparition de fontis en surface uniquement si les galeries sont très peu profondes et si les caractéristiques géomécaniques des grès et des discontinuités naturelles qui les affectent sont très faibles. Dès que la profondeur dépasse 8 mètres, la remontée de la cloche d'éboulement est bloquée avant d'atteindre la surface par l'effet de foisonnement des terrains éboulés. Les Houillères du Bassin de Lorraine s'appuient sur ces résultats pour la mise en sécurité des ouvrages d'infrastructure minière tels que l'ancienne galerie d'exhaure de Falck réalisée au XIX'e siècle.

Mots-clés: stabilité, galerie, mine, grès, remblayage.

\section{Effect on the surface}

of the shallow deep drifts excavated in the Vosges sandstones. Example of the treatment of the old drift falck

The conditions of the stability of the drifts excavated in the Vosges sandstones, that might be modified by overburden weakening and/or flooding of drifts, have been analysed. The effects of potential instabilities on the surface level have also been quantified. In the worst case, the performed analysis predicts formation of a sinkhole at soil surface only if the drifts are very close to the surface and in case the mechanical properties of the sandstones and those of natural discontinuities are very poor. Beyond eight meters of depth, evolution of collapse arch is stopped prior to reaching soil surface, as a matter of dilatancy of collapsed strata. The «Houillères du Bassin de Lorraine " uses these results for safety assessment of the drift belonging to mining infrastructure such as old drift Falck driven in 19th century for water seepage.

Key words: stability, drift, mining, sandstone, backfilling
} 


\section{Introduction}

Dans le cadre de l'exploitation du gisement houiller lorrain, de nombreuses anciennes galeries ont été creusées dans la formation des grès vosgiens à faible profondeur.

Il a été établi que les effets prévisibles en surface dus à d'éventuelles instabilités des galeries minières excavées dans les formations du Houiller sous-jacentes, situées à des profondeurs variant de 80 à $400 \mathrm{~m}$, sont extrêmement faibles (Piguet, 1997). Par contre, les effets sur la stabilité cles terrains de surface de la dégradation de la tenue des galeries creusées à des profondeurs plus faibles dans les grès vosgiens, pourraient être plus importants. A la demande de l'Unité de Gestion de l'Environnement et du Patrimoine Industriel (UGEPI) des Houillères du Bassin de Lorraine (HBL), I'INERIS a évalué et quantifié les effets prévisibles en surface induits par la dégradation à long terme de l'état de stabilité de ces galeries, cette dégradation pouvant être déclenchée ou accélérée par leur ennoyage consécutif à l'arrêt des pompages d'exhaure minière dû à la cessation de l'exploitation.

Dans le cas où la stabilité des galeries n'est pas assurée sur le long terme, des mesures compensatoires doivent généralement être prises pour prévenir les risques liés à la présence de ces ouvrages peu profonds dans le sous-sol de régions parfois fortement urbanisées. C'est le cas notamment de l'ancienne galerie d'exhaure minière de Falck, située sur le territoire de la commune de Falck, à $2 \mathrm{~km}$ à l'est du village de Hargarten-aux-Mines et $3 \mathrm{~km}$ au nord du siège II de La Houve (département de la Moselle). Cette galerie a fait l'objet de travaux de traitement, également décrits dans cette communication, afin de garantir la sécurité et la salubrité publiques ainsi que les caractéristiques essentielles du milieu environnant.

\section{2}

\section{Contextes géologique, géotechnique et minier}

\section{1}

\section{Cadre géographique et contexte géologique}

Le bassin houiller sarro-lorrain s'étend sur une superficie d'environ $140 \mathrm{~km}$ de long sur 70 à $80 \mathrm{~km}$ de large. Il est situé, en partie, au nord-est du département de la Moselle, à $35 \mathrm{~km}$ environ à l'est de Metz et se poursuit au-delà de la frontière allemande (Land de Sarre). Le secteur où se situent les exploitations souterraines présente très schématiquement la forme d'un triangle dont les sommets correspondent approximativement aux villes de Creutzwald au nord, Forbach au nord-est et Faulquemont au sud-ouest (Fig. 1).

Le gisement houiller sarro-lorrain, d'âge carbonifère, est allongé entre le Hunsrück, correspondant à une partie du massif schisteux rhénan, et les Vosges. L'ensemble du gisement forme une série d'anticlinaux et de synclinaux d'orientation générale NE-SW. Le bassin lorrain constitue la partie française de ce gisement.

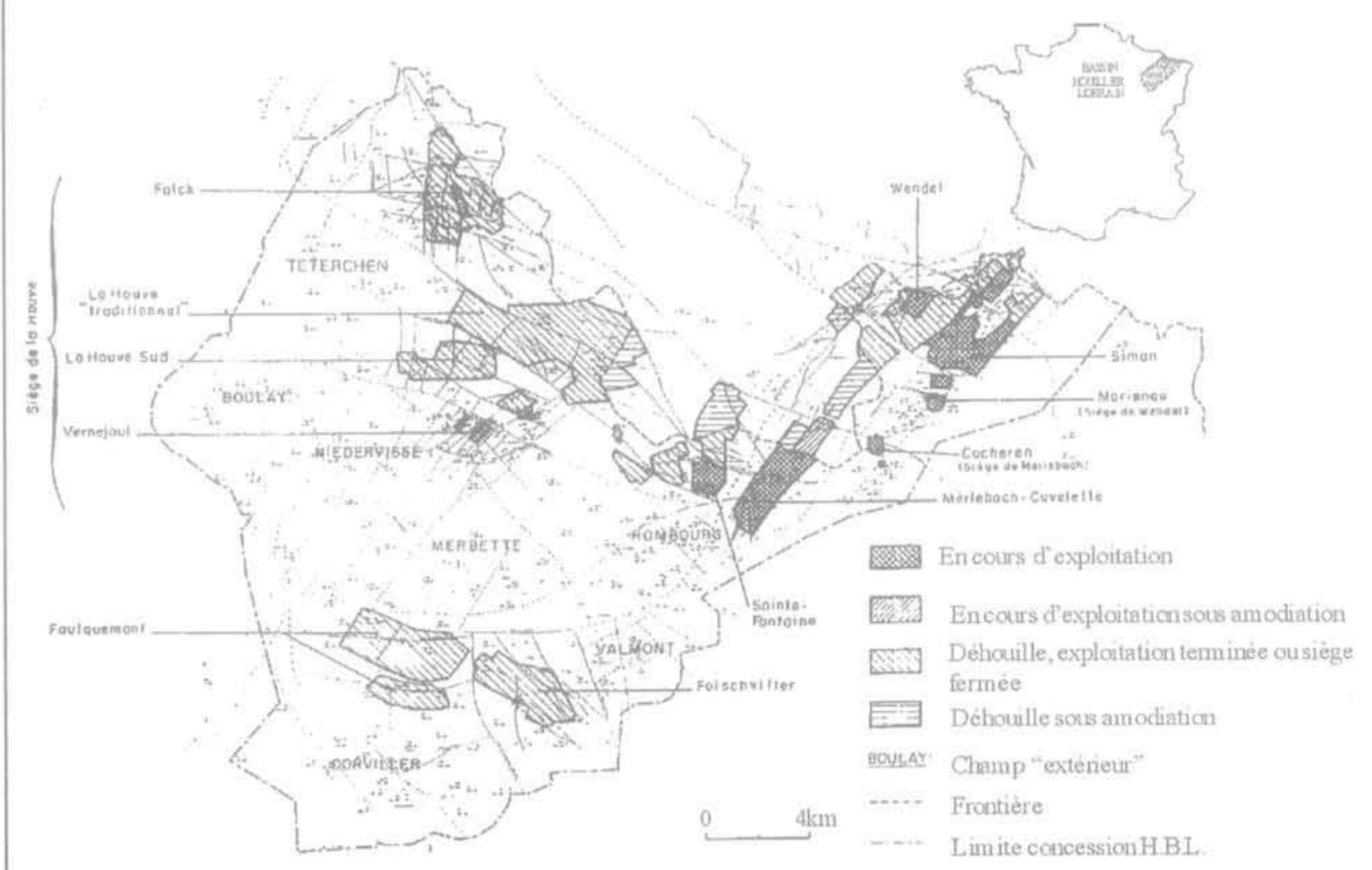

FIG. 1 Situation géographique du bassin houiller lorrain. Location of the Lorraine Collieries. 
Affleurant au nord-est, en Sarre, le Carbonifère s'approfondit vers le sud-ouest sous une couverture de morts-terrains datés du Permien supérieur (grès et conglomérats à ciment carbonaté) et du Trias.

Le Trias inférieur, ou Buntsandstein, est présent dans les Vosges septentrionales et s'étend jusqu'en Allemagne, dans le Palatinat et en Sarre. La série lithographique est constituée, pour l'essentiel, de sables et de grès d'origine fluviatile. Les grès triasiques reposent généralement sur d'autres grès d'âge permien, mais peuvent localement être en discordance sur le socle schistogréseux du Carbonifère (formations du houiller d'âge Stéphanien et Westphalien C). Cette série comporte, de haut en bas (Fig. 2) : la formation des grès bigarrés, d'une puissance de 40 à $50 \mathrm{~m}$, elle-même constituée par les grès à Voltzia, les grès intermédiaires et le conglomérat principal (Buntsandstein supérieur). Les grès bigarrés recouvrent la formation des grès vosgiens, d'une puissance moyenne de l'ordre de $300 \mathrm{~m}$. Buntsandstein moyen). L'importante épaisseur de la formation des grès vosgiens explique, en partie, pourquoi elle a été très fortement exploitée dans des carrières à ciel ouvert, notamment pour la production de matériaux de remblai mis en place par voie hydraulique dans les chantiers miniers. Dans l'ensemble de la formation des grès vosgiens, on distingue généralement deux parties, d'une épaisseur à peu près équivalente de $150 \mathrm{~m}$ chacune

- les grès vosgiens inférieurs encore appelés les couches de Trifels ;

- les grès vosgiens supérieurs encore appelés les couches de Rehberg et Trippstad.

\begin{tabular}{|c|c|c|}
\hline MUSCHE KALKK Suptriaur & $20-40 \mathrm{~m}$ & $\begin{array}{l}\text { Calcaires a Cúratites } \\
\text { Calcairos aEntrocpues }\end{array}$ \\
\hline MESCHE KALK Moycon & $60-70 \mathrm{~m}$ & $\begin{array}{l}\text { Gircupe Anhydrite } \\
\text { Atimes grises } \\
\text { lntacialitions dolom iticpos: }\end{array}$ \\
\hline MUSCHFL KALK Inferivur & $35-40 m$ & 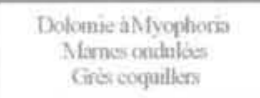 \\
\hline GRESBIGARRES & $40-50 \mathrm{~mm}$ & 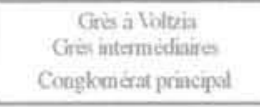 \\
\hline GRES VOSGIENS & $290-300 \mathrm{~m}$ & Nompepturiongaie \\
\hline PERMIIEN & $30-60 \mathrm{~m}$ & \\
\hline STEPHANIEN & $0-500 \mathrm{~m}$ & COVALOUER-TLE HOCTZ \\
\hline WESPHALIENC & Veàne 1 & \\
\hline
\end{tabular}

FIG.1 Log géologique synthétique du Westphalien au Muschelkalk de la région de Faulquemont. Synthetic geological log from Westphalien to Muschelkalk in Faulquemont area.
La partie inférieure est constituée de grès riches en feldspath. La formation montre de fréquentes figures de stratification entrecroisée découpées par de nombreux chenaux. Ces dépôts, conglomératiques à la base, reposent sur les grès d'Annweiler (Buntsandstein inférieur).

Les formations du Buntsandstein (Trias inférieur), à dominante gréseuse et conglomératiques, sont, en général, directement surmontées par les séries carbonatées du Trias moyen (Muschelkalk), d'une épaisseur globale variant de 100 à $150 \mathrm{~m}$, et représentées successivement par : la série des calcaires à cératites et des calcaires à entroques (Muschelkalk supérieur) ; le groupe de l'anhydrite et les marnes grises (Muschelkalk moyen) et, enfin, la dolomie à Myophora, les marnes ondulées et les grès coquilliers (Muschelkalk inférieur).

Sur le plan hydrogéologique, la formation des grès vosgiens est très fortement aquifère puisqu'elle présente une double porosité (de pore et de fissure) avec une perméabilité globale généralement comprise entre $5.10^{-7}$ et $10^{-6} \mathrm{~m} / \mathrm{s}$. Elle constitue un réservoir d'eau potable, souvent d'excellente qualité, fréquemment exploité par sondages. L'alimentation de cette nappe est essentiellement assurée par les précipitations, la piézométrie générale n'indiquant que peu ou pas d'alimentation par infiltration des cours d'eau. Cette piézométrie est, à l'heure actuelle, très fortement influencée par les pompages d'exhaure minière qui engendrent la formation d'entonnoirs (dépressions marquées de la surface piézométrique) et orientent les écoulements vers des points bas. L'arrêt de ces pompages d'exhaure dans les exploitations souterraines engendrera, à terme, une remontée du niveau piézométrique de la nappe des grès. A titre d'exemple, on notera, en termes d'impacts sur la nappe des grès du Trias inférieur des exploitations minières dans le secteur de Faulquemont, que l'exhaure minière a atteint un débit maximum de l'ordre de $1600 \mathrm{~m}^{3} /$ heure. Le rabattement maximal de la nappe des grès vosgiens s'est établi à la cote $+175 \mathrm{~m}$, alors que les estimations effectuées indiquent un niveau piézométrique d'origine, avant travaux miniers, s'établissant aux environs de la cote $+250 \mathrm{~m}$, au toit des grès à Voltzia. Dans ce secteur, la nappe des grès du Trias inférieur était donc vraisemblablement en charge à l'origine.

\section{2}

\section{Principales caractéristiques géotechniques des grès vosgiens}

Les grès vosgiens se situent sur le flanc sud du vaste anticlinal sarro-lorrain orienté NE-SW. Les études effectuées, notamment sur les grès exploités dans les carrières de Merlebach et Centrale, indiquent que le pendage n'excède que rarement une dizaine de degrés vers le SSE. Cet anticlinal est morcelé, selon les cartes géologiques du BRGM correspondant aux feuilles de Forbach et de Boulay, par deux familles de fracturation : l'une majeure et transversale à l'axe du pli, de direction générale NW-SE ; l'autre, de moindre importance, longitudinale à l'axe du pli, orientée globalement NE-SW.

L'ensemble des résultats géotechniques dont on dispose actuellement sur les grès vosgiens provient de plusieurs campagnes de reconnaissance effectuées ces vingt dernières années dans les carrières Simon, Centrale et 
de Merlebach ainsi que pour des études d'ouvrages miniers spécifiques tels que la digue de Carling et le " schistier » de la carrière Simon, Ces données nous permettent de nous faire une idée globale des caractéristiques géomécaniques des grès vosgiens dans la région de Forbach en particulier. En revanche, l'origine fluviatile de ces dêpôts en fait une formation aux variations latérales de faciès parfois très importantes, ce qui complique toute recherche de corrélation entre sondages.

\section{Caractéristiques de cisaillement et pressiométriques}

Dans le cadre de l'étude de la mise en sécurité des fronts de taille de la carrière Simon, trois sondages d'environ $100 \mathrm{~m}$ de profondeur ont été réalisés à quelques mètres du sommet des fronts de taille dont la hauteur peut atteindre $95 \mathrm{~m}$. Les caractéristiques de résistance au cisaillement de différentes couches de grès exploitées ont été estimées à partir d'essais à la boite de Casagrande et par rétro-analyse des incidents observés sur les fronts. Il a été ainsi distingué deux types de terrain

- les terrains assez déconsolidés et peu résistants avec les caractéristiques moyennes suivantes : cohésion de $20 \mathrm{kPa}$ et angle de frottement interne de $40^{\circ}$;

- les terrains plus résistants, avec une cohésion comprise entre 120 et $500 \mathrm{kPa}$, et un angle de frottement interne compris entre $42^{\circ}$ et $62^{\circ}$.

Des essais de cisaillement ont été également réalisés sur les parties indurées du grès exploité dans la carrière de Merlebach. Suite à ces essais, deux couples de caractéristiques de cisaillement ont été retenus: l'un avec une cohésion comprise entre 500 et $600 \mathrm{kPa}$ et un angle de frottement variant de $30^{\circ}$ à $50^{\circ}$ : l'autre avec une cohésion de $80 \mathrm{kPa}$ et un angle de frottement de $48^{\circ}$.

Deux essais pressiométriques pour caractériser les grès situés sous une digue à Carling, donc dans la tranche altérée de surface, ont indiqué, sur une profondeur de 2 à $3 \mathrm{~m}$, des pressions limites allant de 1,4 à $4 \mathrm{MPa}$ pour l'un et de 1 à 1,5 MPa pour l'autre. Au-delà de ces horizons altérés, les pressions limites varient de $3 \mathrm{MPa}$ à plus de $5 \mathrm{MPa}$, valeurs correspondant à un sable très compact. Les valeurs obtenues pour le module pressiométrique sont supérieures ou égales à $30 \mathrm{MPa}$.

Dans l'étude géotechnique pour l'abandon du schistier et du bassin à schlamms de la carrière Simon, pour la tranche de $4 \mathrm{~m}$ d'épaisseur de grès décompacté recouvrant le grès sain, les caractéristiques de cisaillement en contraintes effectives retenues sont les suivantes : cohésion effective de $15 \mathrm{kPa}$ et angle de frottement effectif de $34^{\circ}$.

Toutes ces valeurs indiquent bien que ce grès est sensible aux phénomènes d'altération de surface, ce qui est tout à fait logique au vu de la porosité et de la perméabilité élevées de ce matériau. On retiendra également que la tranche altérée superficielle peut atteindre plusieurs mètres d'épaísseur et possède des caractéristiques géomécaniques comparables à celles d'un sable.

\section{Caractéristiques de dureté et de résistance}

Un ensemble de carottes choisies parmi les plus dures et issues de ces différentes campagnes de son-
Ces essais ont révélé des valeurs movennes de dureté comprises entre :

$-1,5$ et 4 , correspondant à la classe de dureté des roches tendres à assez tendres, pour les carrières de Merlebach ;

- 1 et 2, correspondant à la classe de dureté des roches tendres, pour les carrières Centrale et Simon.

Plusieurs essais mécaniques ont été effectués en laboratoire sur les carottes d'un sondage réalisé très récemment à proximité du puits Wendel 3. Les valeurs de la résistance à la compression simple et à la traction indirecte sont respectivement comprises entre $31,0 \mathrm{MPa}$ et $46,6 \mathrm{MPa}$, et entre $1,5 \mathrm{MPa}$ et $3,3 \mathrm{MPa}$. Un seul des échantillons testés présentait de bien meilleures caractéristiques avec une résistance à la compression uniaxiale de l'ordre de $108 \mathrm{MPa}$ et une résistance à la traction directe de $9,2 \mathrm{MPa}$.

On dispose également de résultats d'une caractérisation mécanique réalisée sur un grès très altéré prélevé dans la carrière de Merlebach. Des essais de compression uniaxiale et triaxiale ont ainsi permis de déterminer les caracteristiques suivantes: E (module $\mathrm{d}^{\prime}$ Young $)=300 \mathrm{MPa} ; \mathrm{R}$ (résistance à la compression

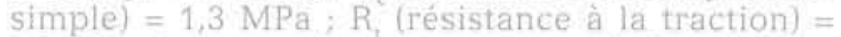
$0,3 \mathrm{MPa}$. On notera également qu'une légère pression de confinement suffit à augmenter très significativement le module d'élasticité et le seuil de plastification du matériau. Les caractéristiques des Grès Vosgiens sont, en général, bien meilleures lorsqu'il n'est pas altéré. F. Homand (1985) signale en particulier les valeurs suivantes: $E=12800 \mathrm{MPa}$ et $R_{c}=56 \mathrm{MPa}$.

\section{Conclusion}

Il ressort de l'ensemble de ces résultats que les grès vosgiens dans la région de Forbach se caractérisent par une alternance de bancs durs et de bancs beaucoup moins indurés, dont les caractéristiques géomécaniques se rapprochent, pour certains niveaux, de celles d'un sable. Compte tenu des variations latérales de faciès très importantes au sein de la formation des grès vosgiens, ces bancs ont une continuité horizontale limitée. Ces niveaux de sable peu consolidé peuvent se situer en surface comme au sein du massif mais sont néanmoins difficilement repérables. De manière très générale, on peut aussi constater que les grès vosgiens de Merlebach possèdent de meilleures caractéristiques mécaniques que ceux de Forbach. Enfin, les grès vosgiens restent un matériau très sensible aux phénomenes d'érosion et d'altération météorique. De ce fait, la tranche altérée de surface, de quelques mètres d'épaisseur, constitue un horizon gréseux peu cohèrent.

3

\section{Analyse des effets de surface induits par les galeries creusées dans les grès vosgiens}

\section{1}

\section{Description géométrique des galeries}

Deux configurations géométriques de galeries creusées dans les grès vosgiens ont été retenues pour l'ana- 
lyse des conditions de leur stabilité : des galeries quadrangulaires de $2 \mathrm{~m}$ de hauteur et de 2,5 $\mathrm{m}$ de largeur ( $5 \mathrm{~m}^{2}$ de section) et des galeries en forme de fer à cheval de $2,4 \mathrm{~m}$ de hauteur maximale et $4 \mathrm{~m}$ de largeur ( $8 \mathrm{~m}^{2}$ de section moyenne). Pour se mettre dans les conditions les plus défavorables, il a également été supposé, qu'à long terme, le soutènement mis éventuellement en place lors de leur creusement ne joue plus aucun rôle. Enfin, ces galeries sont situées à des profondeurs variant de 5 à $80 \mathrm{~m}$.

\section{2}

\section{Origine et nature des effets de surface}

Les grès vosgiens sont plutôt considérés comme étant des roches relativement homogènes et résistantes, Les galeries creusées dans ces grès ne doivent donc pas, a priori, connaitre de problème dé stabilité mécanique mème lorsqu'elles sont proches de la surface, à moins d'une dégradation locale particulièrement importante de leurs caractéristiques géomécaniques. Parmi les facteurs susceptibles de contribuer à une réduction significative de leurs caractéristiques mécaniques en grand, on retiendra en particulier :

- la diminution de l'épaisseur moyenne des bancs : les observations géologiques (BRGM, 1976) montrent que l'épaisseur des bancs de grès est généralement assez importante $(0,5 \mathrm{~m}$ à $6 \mathrm{~m})$. Une diminution locale de cette épaisseur peut favoriser la rupture des bancs du bas-toit de la galerie :

- l'augmentation de la densité de fracturation des bancs: les bancs sont généralement très peu fracturés mais la densité de fracturation peut augmenter de manière importante à proximité de grandes discontinuités majeures (failles) :

- la diminution de la résistance mécanique du grès, qui peut être liée à des phénomènes de vieillissement (altération météorique et/ou en présence d'eaux chimiquement agressives par exemple), de fatigue du matériau ou à une variation de leur teneur en eau.

J.-P. Piguet (1997) a montré que les effets de surface induits par une galerie située à une profondeur supérieure à $80 \mathrm{~m}$ (limite des terrains houillers) étaient toujours liés à un affaissement progressif des terrains susjacents mais son amplitude est quasiment négligeable compte tenu de la largeur L des galeries rapportée à leur profondeur $\mathrm{H}$ (ratio $\mathrm{L} / \mathrm{H}$ inférieur à 0,05 ). Par contre, dans le cas de galeries très peu profondes, creusées dans les grès vosgiens altérés, la possibilité d'un effondrement de type fontis doit être envisagée (Piguet et Wojtkowiak, 2001).

Les fontis se développent préférentiellement au surplomb de carrefours de galeries car ce sont les endroits où la portée du toit non soutenue est la plus importante et les risques d'éboulement du toit les plus élevés. L'apparition d'un fontis à l'aplomb d'une galerie isolée peu profonde est toutefois possible dans le cas d'un recouvrement très dégradé et/ou altéré. Les conséquences de l'apparition d'un fontis en surface sont souvent d'extension relativement limitée (quelques mètres à quelques dizaines de mètres au maximum) mais peuvent s'avérer très graves si elles se localisent sous une construction ou un ouvrage d'infrastructure (Vachat, 1982).

Le coefficient de foisonnement des terrains $\mathrm{K}$ (volume des terrains effondrés rapporté à leur volume initial) peut jouer un rôle prépondérant dans l'évolution des montées de voûte au toit des galeries. Ainsi, lorsque les terrains éboulés ont un coefficient de foisonnement élevé, même en présence de conditions de stabilité très défavorables (terrains très peu résistants), le fontis ne débouchera pas forcément au jour du fait de l'auto-comblement du vide souterrain induit par le foisonnement des terrains (Wojtkowiak et Didier, 1999). Un calcul simple, classiquement utilisé pour estimer la hauteur de toit susceptible de s'ébouler (notamment pour le cas des exploitations avec foudroyage) consiste à écrire $: h+h=K \cdot h$, avec $h$ la hauteur de la cavité et h. la hauteur de la partie effondrée. On vérifie facilement avec cette formule que pour des exploitations foudroyées, la hauteur de la cloche varie de 10 fois à 2 fois l'ouverture de la couche exploitée, si les valeurs du coefficient de foisonnement $\mathrm{K}$ varient respectivement de 1,1 à 1,5 . Ceci signifie que la hauteur $h$, pour une cavité d'ouverture moyenne $2,5 \mathrm{~m}$ par exemple, varie de $5 \mathrm{~m}$ à $25 \mathrm{~m}$.

Notons qu'au-dessus de la cloche d'éboulement, dans les terrains stratifiés, les bancs fléchissent et s'appuient sur les blocs éboulés. Cette flexion induit une fissuration mécanique et l'ouverture des plans de discontinuité naturelle existant au sein de ces bancs (diaclases, plans de stratification), mais ceux-ci gardent encore une certaine continuité géométrique d'ensemble (Mahmoud, 1984)

\section{3}

\section{Facteurs aggravants}

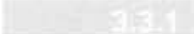 \\ Influence de l'eau et du temps}

La présence d'eau peut aussi avoir une influence sur les propriétés mécaniques des terrains de recouvrement et donc sur la stabilité mécanique des ouvrages. Une augmentation de la teneur en eau du matériau, sous l'effet des infiltrations d'eau superficielle ou du battement du niveau de la nappe phréatique, peut altérer la résistance mécanique des roches. Ainsi, en passant de l'état sec à l'état saturé, les grès perdent environ $40 \%$ de leur résistance á la compression uniaxiale (Pineau, 1976).

Dans certains cas, la pression d'eau dans les joints de stratification peut également contribuer à faire fléchir les dalles du toit jusqu'à leur rupture (Maury, 1979).

D'autre part, la résistancé de certaines roches semble décroître de manière significative en fonction du temps. Il est donc nécessaire de prendre en compte le concept de résistance ultime, ou résistance admissible à long terme, pour évaluer l'évolution de la stabilité d'une cavité dans le temps.

\section{Actions anthropiques}

Les phénomènes d'origine anthropique, en modifiant les conditions mécaniques et hydrauliques qui règnent au sein du massif dans un sens pratiquement toujours défavorable, interviennent en tant qu'initiateurs ou accélérateurs des processus de dégradation. Les principales perturbations de ce type sont les suivantes: 
- les surcharges pondérales, dues à la construction de nouveaux bâtiments ou d'ouvrages d'art, créent des surcontraintes locales qui peuvent se reporter sur les épontes des cavités souterraines ;

- la circulation d'engins de fort tonnage en surface peut être à l'origine d'une fatigue des matériaux suite à des cycles successifs de chargement/déchargement (Bertrand et al., 1981)

- les modifications du régime hydraulique et d'écoulement des eaux souterraines au sein du massif liées, par exemple, à l'arrêt des pompages d'exhaure minière.

\section{4}

\section{Stabilité à long terme des galeries creusées dans les grès vosgiens}

L'analyse des conditions de stabilité à long terme des galeries creusées dans les grès vosgiens, avant, pendant et après leur ennoyage, a été effectuée à partir des résultats de simulations sur modèle numérique (Thoraval, 1997). Les calculs ont été réalisés à l'aide des codes numériques UDEC (Universal Distinct Element Code) et FLAC (Fast Lagrangian Analysis of Continua). Ces codes permettent de simuler le comportement hydromécanique d'un milieu rocheux à double porosité (de pore et de fracture).

Pour simuler le vieillissement du grès, nous avons considéré, hypothèse jugée extrêmement pessimiste, que le grès avait partout les caractéristiques mécaniques de celui exploité dans la carrière de Freyming $\left(E=300 \mathrm{MPa} ; \mathrm{R}_{c}=1,3 \mathrm{MPa} ; \mathrm{R}_{\mathrm{c}}=0,3 \mathrm{MPa}\right)$. Ces caractéristiques proviennent d'essais standard réalisés dans des conditions hydriques non contrôlées. Compte tenu de la variabilité des données, il nous a semblé pertinent d'opter ici pour une approche sécuritaire qui rend inutile la distinction entre les propriétés drainées (valeurs à utiliser pour un calcul hydromécanique avec FLAC) ou non drainées (valeurs à utiliser pour un calcul hydromécanique avec UDEC).

Les valeurs des caractéristiques choisies pour les joints de stratification (indicées j) et les fractures (indicées f) sont les suivantes: raideur normale: $\mathrm{k}_{\mathrm{nl}}=100 \mathrm{MPa} / \mathrm{m}, \mathrm{k}_{\mathrm{n}}=1000 \mathrm{MPa} / \mathrm{m}$; raideur tangentielle : $\mathrm{k}_{\mathrm{si}}=10 \mathrm{MPa} / \mathrm{m}, \mathrm{K}_{\mathrm{vf}}=100 \mathrm{MPa} / \mathrm{m}$. Les discontinuités obéissent à une loi de comportement élasto-plastique parfait définie par les paramètres suivants : anqle de frottement: $\varphi=\varphi_{\mathrm{f}}=10^{\circ}$; cohésion: $\mathrm{c}=0 \mathrm{MPa}, \mathrm{c}_{\mathrm{f}}=0,01 \mathrm{MPa}$; résistance à la traction: $\mathrm{R}_{\mathrm{t}}=0 \mathrm{MPa}, \mathrm{R}_{\mathrm{If}}=0,01 \mathrm{MPa}$.

Trois configurations géologiques très différentes, qui rendent compte de la variabilité spatiale de la structure et de la fracturation au sens large des grès vosgiens, ont été distinguées :

- configuration $n^{\circ} 1$ : le recouvrement de la galerie est constitué d'un grès massif, c'est-à-dire ni stratifié ni fracturé, configuration bien sûr la plus favorable des trois à la stabilité des galeries :

- configuration $n^{\circ} 2$ : les terrains gréseux, cette fois-ci, sont nettement stratifiés en bancs d'épaisseur variable. Trois épaisseurs de banc ont été prises en compte : $0,25 \mathrm{~m}, 0,5 \mathrm{~m}$ et $1,00 \mathrm{~m}$. Comparées à celles observées sur le terrain, ces épaisseurs de banc sont considérées comme faibles, voire très faibles, ce qui nous place du côté de la sécurité pour notre analyse puisque le risque de rupture du banc diminue lorsque son épaisseur aug-
- configuration $n^{\circ} 3$ : la galerie est traversée par une zone faillée. Dans ce cas, le recouvrement perd localement de sa cohésion. On peut alors l'assimiler à un milieu constitué de blocs de petites dimensions. Deux tailles moyennes de bloc ont été retenues dans notre analyse effectuée à deux dimensions (2D) : $1 \mathrm{~m}^{2}$ et $0,25 \mathrm{~m}^{2}$. Ces dimensions sont également faibles en regard des observations faites sur le terrain. Notons que nous avons utilisé une commande spécifique du logiciel UDEC, qui permet de générer des géométries plus réalistes que celles consistant, par exemple, à découper le grès en deux familles de fractures régulièrement espacées.

Par ailleurs, nous avons fait varier la profondeur de la galerie entre 5 et 80 mètres.

\section{1}

\section{Conditions de stabilité avant l'arrêt des pompages d'exhaure minière}

A partir des résultats obtenus, on a pu établir que, si les galeries restent hors d'eau :

- les effets en surface d'une éventuelle dégradation des conditions de stabilité des galeries sont négligeables lorsque celles-ci sont creusées dans des bancs de grès épais et peu fracturés (configuration 1), et ce, même lorsqu'elles sont situées à quelques mètres de profondeur seulement. Ainsi, dans la gamme de profondeur considérée, l'affaissement de la surface du sol n'excède pas 1,2 mm pour des galeries quadrangulaires et $2 \mathrm{~mm}$ pour les galeries en forme de fer à cheval. Le ratio " affaissement/convergence du toit de la galerie », qui traduit l'effet d'amortissement des mouvements de terrain dans le recouvrement, diminue de façon quasi hyperbolique avec la profondeur des galeries. Les valeurs de pente et de déformation maximale calculées diminuent également bien sûr avec la profondeur des galeries. Pour les galeries les moins profondes, on obtient une valeur de déformation de $4.10^{-5}$ pour les galeries rectangulaires et de $2.10^{-4}$ pour la forme en fer à cheval

- dans le cas de grès très stratifiés (configuration 2), les effets en surface d'une éventuelle dégradation du recouvrement sont légèrement amplifiés par rapport au cas précédent tout en restant négligeables. Les déplacements et les affaissements induits sont inversement proportionnels à l'épaisseur moyenne des bancs de grès. Ainsi, dans le cas d'une galerie quadrangulaire, I'amplitude de l'affaissement est respectivement de $6 \mathrm{~mm}, 11 \mathrm{~mm}$ et $17 \mathrm{~mm}$ lorsque l'épaisseur moyenne des bancs est égale à $1 \mathrm{~m}, 0,5 \mathrm{~m}$ et $0,25 \mathrm{~m}$. Les valeurs de pente et de déformation horizontale maximale augmentent de façon significative lorsque la stratification des grès est de plus en plus marquée. Ceci dit, même dans le cas extrême d'un litage fin des grès, les effets potentiels en surface restent faibles :

- lorsque les galeries traversent des zones faillées (configuration 3), on pouvait s'attendre à ce que les risques d'instabilité au fond puis d'apparition de fontis à la surface soient plus importants du fait d'une plus grande densité de fracturation des grès. Or, si cette fracturation n'est pas trop importante, la hauteur de la cloche d'éboulement qui se forme au toit de la galerie reste faible, ainsi que les effets ressentis à la surface. Si la fracturation des terrains de recouvrement est plus intense, l'apparition d'un fontis en surface n'est possible 
que si les galeries sont très proches de la surface et si les caractéristiques de cisaillement des fractures qui affectent les grès sont très faibles. Dès que l'épaisseur du recouvrement dépasse $8 \mathrm{~m}$, la montée de la cloche d'éboulement est stoppée avant d'atteindre la surface par l'effet de foisonnement des terrains ${ }^{(7)}$ éboulés induisant ainsi l'auto-comblement du vide souterrain (Fig. 3).

Il faut se rappeler que ces résultats ont été obtenus avec des caractéristiques géomécaniques des grès très faibles, correspondant à des grès très altérés, donc défavorables à la stabilité des galeries. De plus, l'ensemble des calculs est basé sur un angle de frottement des discontinuités de $10^{\circ}$. En réalité, on sait que la rugosité des épontes des fractures contribue à augmenter de façon très significative la valeur de l'angle de frottement jusqu'à des valeurs de 30 à $40^{\circ}$. Une très forte influence de ce paramètre sur la stabilité du toit immédiat de la galerie a été mise en évidence. Ainsi, d'après le modèle, un angle de $15^{\circ}$ suffit à éviter la formation d'un fontis (l'éboulement du toit s'interrompt bien avant d'atteindre la surface) et une valeur de $20^{\circ}$ réduit de façon significative l'importance de l'éboulement du toit (la cloche d'éboulement au toit de la galerie a, dans ce cas, moins d'un mètre de hauteur).

\section{9}

\section{Stabilité à long terme des galeries pendant et après leur ennoyage}

La remontée du niveau piézométrique de la nappe aquifère consécutive à l'arrêt des pompages d'exhaure, a été simulée depuis la base du modèle (c'est-à-dire à $50 \mathrm{~m}$ de profondeur sous le mur de la galerie) jusqu'à la sur-

\footnotetext{
"Le fogiciel UDEC permet de simuler la chute des blocs de leur position initiale dans le recouvrement jusqu'à une nouvelle position stable au mur de la galerie, Les blocs instables se ré-agencent en laissant des vides entre eux. L'apparition des vides entre les blocs permet d'approcher explicitement le phénomène de foisonnement des terrains.
}

face du sol. On suppose donc que la galerie joue tout d'abord le rôle d'un drain parfait. Dans ce cas, la pression d'eau dans la galerie $P_{\text {tal }}$ est égale à zéro. Nous avons simulé ensuite une augmentation de la pression d'eau dans la galerie, traduisant ainsi son ennoyage progressif, jusqu'à ce que la cote du niveau d'eau coincide avec celle de la surface du sol. Notons que cette hypothèse est très pessimiste car la grande majorité des ouvrages (près de $95 \%$ ) est et restera hors d'eau. D'autre part, les HBL prendront des mesures compensatoires pour éviter l'affleurement de la nappe dans les endroits où ce phénomène pourrait éventuellement se produire.

\section{Alquit \\ Cas d'un massif peu ou pas fracturé}

Dans ce cas, le grès est supposé perméable. Les caractéristiques physiques et hydrauliques choisies pour la matrice rocheuse sont les suivantes : $p$ (poids volumique du fluide) $=10 \mathrm{kN} / \mathrm{m}^{3}$; n (porosité de pore) $=0,2$ et $\mathrm{k}=10^{-5} \mathrm{~m} / \mathrm{s}$ (conductivité hydraulique) ${ }^{12}$.

La variation des conditions hyciriques (ennoyage) n'a pas été accompagnée, dans le modèle, d'une réduction supplémentaire des caractéristiques mécaniques des grès ${ }^{(3)}$. En effet, il a été supposé qu'elle était déjà implicitement prise en compte par notre choix très pessimiste d'introduire dans les modèles les résultats de la caractérisation géotechnique réalisée sur les grès très altérés de la carrière de Freyming.

\footnotetext{
13: La valeur de perméabilité choisie ici est un peu supérieure à la valeur moyenne généralement mesurée (voir 5 2.1). Si ce choix peut modifier les vitesses de circulation de l'eau dans la matrice poreuse, il a peu d'effet sur les valeurs des pressions interstitielles, donc sur la stabilité de la galerie.

(19. Le grès se désature pendant la période d'exploitation du fait de l'exhaure minière puis se resature progressivement au fur et à mesure de la remontèe des eaux. Il est possible que cette resaturation induise une réduction de la résistance des grès vosgiens comme cela est constaté pour d’autres grès et matériaux tels que le minerai de fer lorrain et la craie.
}

Profondeur $10 \mathrm{~m}$

Profondeur $5 \mathrm{~m}$
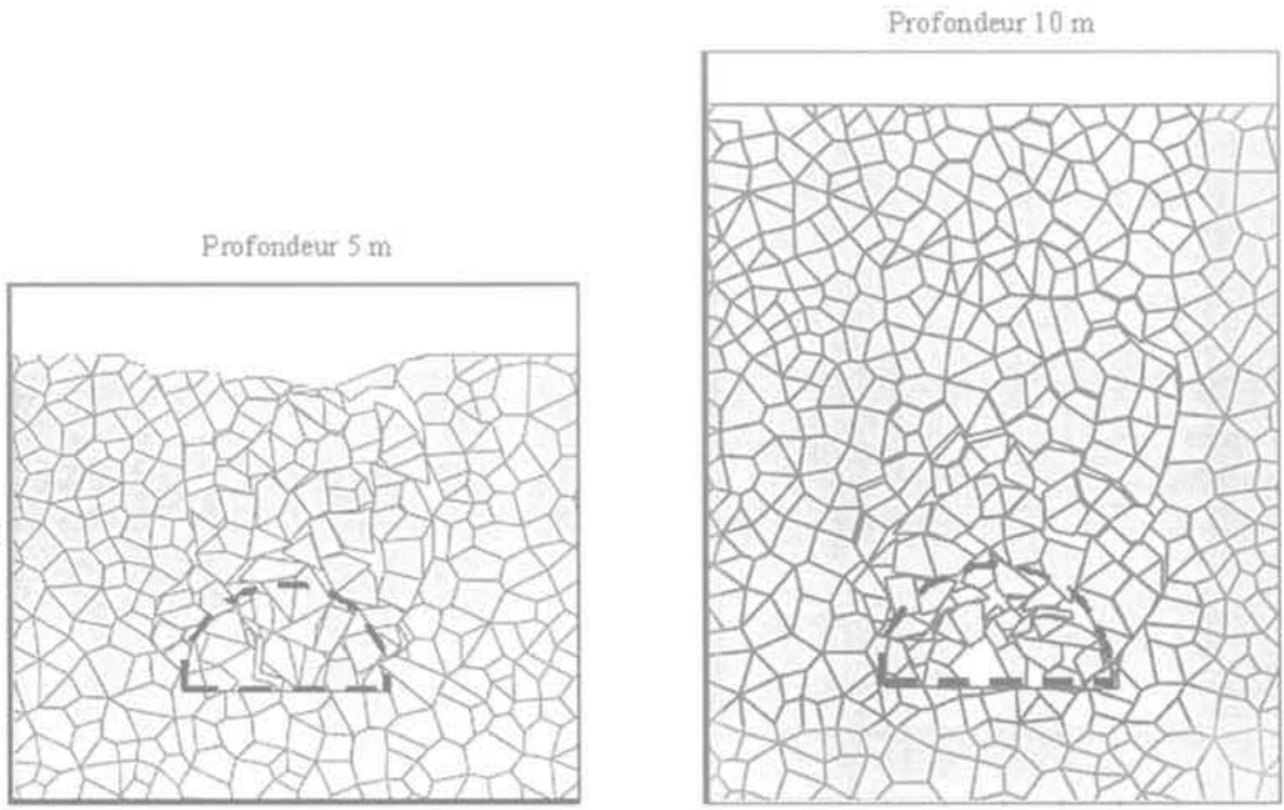

FIG. 3 Comportement des terrains dans les zones de faille en fonction de la profondeur (Y'effondrement s'interrompt avant d'atteindre la surface pour une galerie à $10 \mathrm{~m}$ de profondeur).

Depth effect on the rockmass behavior above the gallery in the fault zones (collapse stops before reaching surface for a gallery at $10 \mathrm{~m}$ depth). 
L'analyse de l'évolution, suite à la remontée des eaux souterraines, des conditions de stabilité d'une galerie creusée dans un massif peu ou pas fracturé (configuration $n^{\circ} 1$ ) est complexe du fait de la multiplicité des effets induits par l'eau. Lorsque le grès est saturé en eau à une pression $\mathrm{P}$, la relation contrainte/déformation fait intervenir le tenseur des contraintes effectives $\left[\sigma^{\prime}\right]$ qui est lié au tenseur des contraintes totales $[\sigma]$ par la relation de Terzaghi $[\sigma]=$ $[\sigma]$ - P [I], où P est la pression de l'eau et I le tenseur identité. En représentant les contraintes principales dans le plan de Mohr, on constate que le cercle de Mohr correspondant aux contraintes effectives se rapproche de la courbe intrinsèque au-delà de laquelle l'état de contrainte conduit à la rupture, Le risque de rupture est d'autant plus important que l'état de contrainte avant ennoyage est proche de la rupture (à proximité de la galerie notamment)

Dans le cas d'une galerie quadrangulaire située à $20 \mathrm{~m}$ de profondeur et qui s'ennoie proqressivement jusqu'à ce que la cote piézométrique atteigne la surface du sol, on constate, à partir des résultats des simulations effectuées, que l'ennoyage à pour effet d'augmenter la contrainte verticale totale. Cette auqmentation reste cependant limitée (de $0,48 \mathrm{MPa}$ à $0,52 \mathrm{MPa}$ pour un point situé à $4 \mathrm{~m}$ du parement de la galerie) du fait de la possibilité du milieu de se déformer. On observe en effet que la remontée de la nappe induit des convergences millimétriques dans la galerie, mais l'essentiel des déformations se traduit par une remontée globale des terrains provoquant un soulèvement global de la surface du sol d'environ $60 \mathrm{~mm}$. Cette valeur correspond environ à un soulèvement de $0,8 \mathrm{~mm}$ par mètre de remontée du niveau de la nappe (on a modélisé ici une remontée de la nappe de $50 \mathrm{~m}$ au-dessous du mur de la galerie jusqu'à la surface).

Pour des profondeur's de 5, 10 et $20 \mathrm{~m}$, le massif environnant la galerie conserve un comportement globalement élastique. Pour une profondeur de $30 \mathrm{~m}$, des zones plastiques se développent autour de l'ouvrage lorsque la nappe remonte environ $15 \mathrm{~m}$ au-dessus de la galerie. Les parements se dégradent mais les effets de cette dégradation ne sont quasiment pas perçus en surface du fait de la profondeur de la galerie et parce qu'ils sont gommés par le soulèvement global des terrains induit par la remontée des eaux au sein du massif gréseux

\section{Cas d'un massif de grès stratifié ou très fracturé}

Dans le cas d'un massif stratifiè, on considère que l'essentiel des écoulements se fait dans les joints de stratification et les discontinuités naturelles. En conséquence, la matrice rocheuse est assimilée, cette fois-ci, à un milieu imperméable. Les propriétés hydrauliques retenues pour les fractures sont les suivantes : ouverture initiale des fractures $a=1 \mathrm{~mm}$, variant dans une gamme de $0,2 \mathrm{~mm}$ (ouverture minimale) à $5 \mathrm{~mm}$ (ouverture maximale) au cours des calculs : conductivité hydraulique (dans les fractures) : $\mathrm{k}=2,8 \cdot 10^{-6} \mathrm{~m} / \mathrm{s}$.

Les joints de stratification sont supposés obéir à une loi de comportement élastoplastique avec un critère de plasticité de Mohr-Coulornb. L'introduction d'une pression d'eau dans la fracture conduit à la modification du critère de plasticité (dans le sens d'une diminution de la cohésion des joints et de leur angle de frottement). Les valeurs choisies étant déjà très pessimistes $(c=$ $\left.0,01 \mathrm{MPa} ; \mathrm{Rt}=0,01 \mathrm{MPa} ; \varphi=10^{\circ}\right)$, nous n'avons pas simulé de réduction supplémentaire de ces caractéristiques.

Par rapport au cas étudié précédemment, les effets supplémentaires induits par l'eau sont les suivants

- l'eau présente dans les joints de stratification exerce une pression P sur les bancs de grès. Cette pression tend à augmenter légèrement les valeurs des contraintes s'exerçant dans ces bancs. Compte tenu des conditions aux limites imposées sur les faces du modèle (déplacement vertical nul à la base du modèle, déplacement horizontal nul sur les faces latérales), les bancs tendent à se déplacer vers la surface. Les déformations induites sont d'environ $1 \mathrm{~mm}$ par mètre de couverture de grès

- la pression d'eau $\mathrm{P}$ dans la fracture déplace le point $\left(\sigma_{n}, \tau\right)$, où $\sigma_{n}$ est la contrainte normale du joint et $\tau$ la contrainte de cisaillement vers le point $\left(\sigma^{\prime}, \tau\right)$ dans le plan de Mohr-Coulomb avec $\sigma^{\prime}=\sigma-$ P. Si la pression P est suffisamment forte, le point peut se déplacer jusqu'au critère et ainsi provoquer une plastification du joint

- la simulation hydromécanique réalisée pour les grès stratifiés obéit au mème phasage que pour les grès massifs à savoir une simulation de la remontée de la nappe jusqu'à la surface, puis une augmentation progressive de la pression d'eau dans la galerie. Cette dernière agit comme un confinement qui réduit le risque de flambage des bancs au mur et au toit de la galerie.

Nous allons à présent illustrer ces différents effets pour une galerie quadrangulaire creusée à $20 \mathrm{~m}$ de profondeur dans un massif stratifié constitué de bancs de grès de 0,5 m d'épaisseur moyenne. Nous avons suivi l'évolution de l'ouverture des joints de stratification au fur et à mesure de la remontée de la nappe. Après creusement de la galerie, l'ouverture est de $3 \mathrm{~mm}$ au toit et de 4 mm au mur. Lorsque la galerie est drainante, on observe des contrastes de pression importants de part et d'autre des premiers bancs de grès supposés imperméables. Cette pression a pour effet d'augmenter l'ouverture des bancs jusqu'à $12 \mathrm{~mm}$ au toit et $16 \mathrm{~mm}$ au mur. Lorsque la galerie est noyée, la pression $\mathrm{P}_{\text {. }}$ augmente progressivement et vient exercer un confinement sur les parois de la galerie. Ce phénomène se traduit par une réduction des ouvertures des bancs de $3 \mathrm{~mm}$ au toit et de $6 \mathrm{~mm}$ au mur.

La mise en eau représente donc une phase critique du point de vue de la stabilité dé la galerie. Cette phase coincide avec une période oủ la pression d'eau dans la galerie est faible devant celle qui s'exerce dans les joints de stratification au toit et au mur immédiats.

L'amplitude du soulèvement des terrains, lié à la remontée des eaux, précédemment observé, augmente avec la densité des joints de stratification : $1,6 \mathrm{~mm} / \mathrm{joint}$ dans le cas de banc de $1 \mathrm{~m} ; 1 \mathrm{~mm} / \mathrm{joint}$ dans le cas de banc de $0,25 \mathrm{~m}$ soit $4 \mathrm{~mm} / \mathrm{m}$. Ce soulèvement est peu affecté par la présence des galeries dans le cas d'une stratification d'épaisseur supérieure à $0,5 \mathrm{~m}$. Par contre, lorsque l'épaisseur des bancs est très faible $(\mathrm{e}=0,25 \mathrm{~m})$, la déformation des terrains autour de la galerie augmente et induit un léger différentiel de déplacement vertical pendant la phase transitoire (galerie drainante).

La mise en pression des joints de stratification induit une séparation, voire la rupture des bancs. Cette rupture est constatée presque systématiquement au toit et au mur lorsque l'épaisseur des bancs est de 0,25 m. 
On l'observe également pour un litage de $0,5 \mathrm{~m}$ dans le cas des galeries « fer à cheval » situées à des profondeurs supérieures à $10 \mathrm{~m}$. Aucune rupture n'est observée jusqu'à des profondeurs de $20 \mathrm{~m}$ dans le cas de bancs de $1 \mathrm{~m}$ d'épaisseur.

Lorsqu'un banc se rompt, les cassures verticales apparaissent vers l'intérieur de la galerie (Fig. 4). La largeur du toit ou du mur diminuant à ce niveau, les ruptures ne sé propagent pas au deuxième banc (aucune rupture du deuxième banc n'a été constatée pour les cas traités). Jusqu'à une profondeur de $20 \mathrm{~m}$, on peut donc constater qu'il n'y a pas de progression de la rupture dans le haut-toit. Notons que, même si ce phénomène était observé pour des profondeurs supérieures. les conséquences en surface de ces dégradations resteraient trés faibles.

Dans le cas des zones très fracturées ou faillées, on considère également que les blocs sont imperméables et que les écoulements se produisent essentiellement dans le réseau de fractures.

Nous avons vu que pour des profondeurs relativement faibles (inférieures à $10 \mathrm{~m}$ ) et sans eaur, le toit des galeries s'éboule en formant une cloche d'éboulement. Si la fracturation est très importante et affecte des terrains de très faible résistance au cisaillement, des galeries situées à $5 \mathrm{~m}$ de profondeur peuvent donner naissance à la formation de fontis en surface. On peut légitimement se demander si, suite à la remontée de la nappe, on n'observe pas d'instabilités mécaniques supplémentaires au fond puis en surface.

Dans le cas où la fracturation découpe des blocs d'une taille de $1 \mathrm{~m}^{2}$, la remontée des eaux induit pour certaines configurations un détachement de blocs préalablement stables des parements de la cavité constituée par la galerie et la cloche d'éboulement. Le mouvement de ces blocs ne dépasse pas une dizaine de centimètres car la cavité est déjà partiellement remblayée par des blocs tombés préalablement à la remontée des eaux. On n'observe donc quasiment aucun effet en surface de cette dégradation Dans le cas d'une fracturation plus dense (blocs de $0,25 \mathrm{~m}^{2}$ ) et de galeries situées à quelques mètres de profondeur seulement, on note que les fontis apparus précédemment sont réactivés et se tassent légèrement de quelques centimètres. Aucune évolution significative n'est observée dans les autres cas, mis à part un léger soulèvement des terrains (Fig. 5).

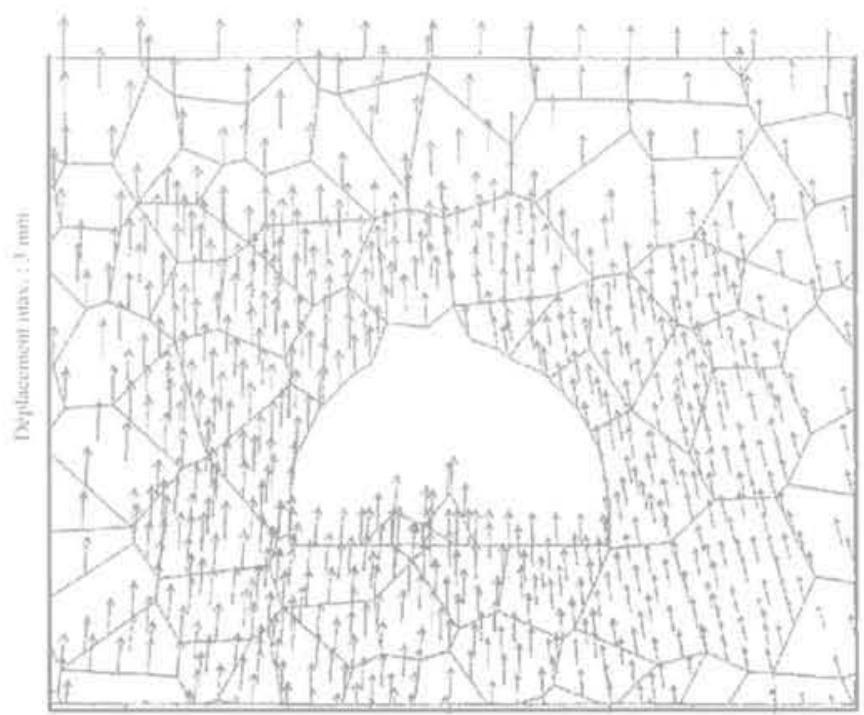

FIG 5 Soulèvement induit par la remontée des eaux pour une galerie à $5 \mathrm{~m}$ de profondeur.

Surface uprising induced by the water table increase for a gallery at $5 \mathrm{~m}$ depth.

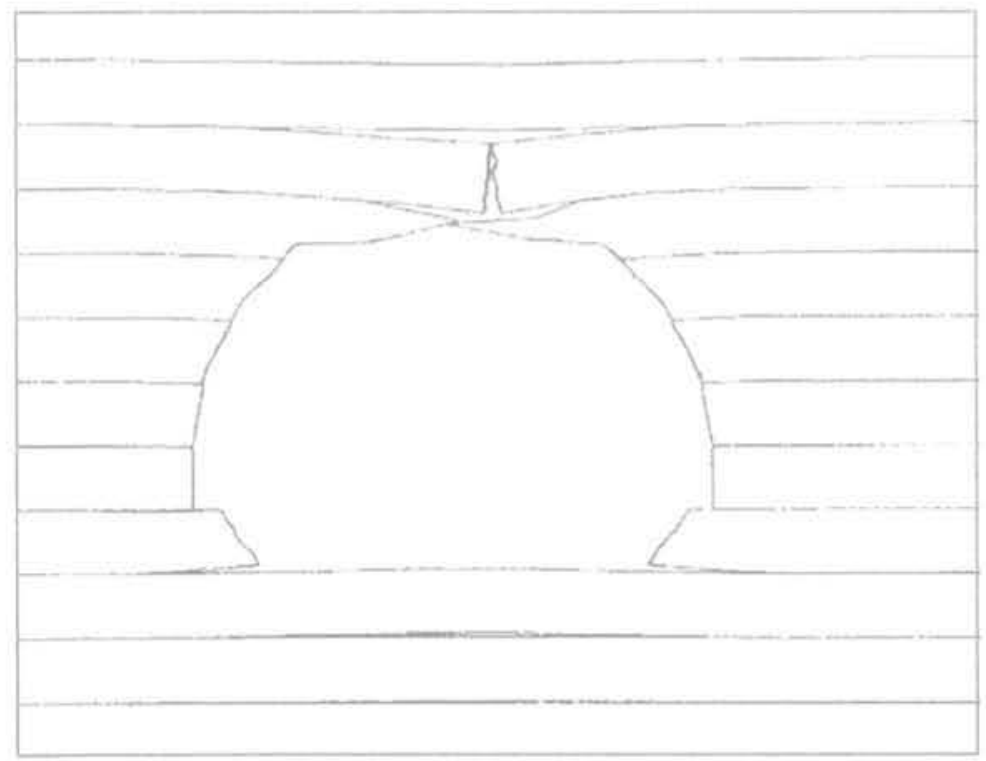

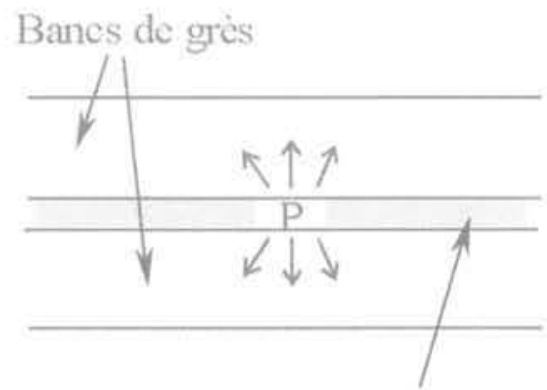

Joint de stratification

FG, 4 Rupture des bancs du toit et au mur des galeries due à la mise en pression de joints de stratification (pour une galerie de $20 \mathrm{~m}$ de profondeur).

Strata failure at the gallery roof and wall due to hydraulic pressure (for a gallery at $20 \mathrm{~m}$ depth). 


\section{Synthèse des résultats}

Le premier objectif de l'étude réalisée était d'analyser les conditions de stabilité de galeries minières creusées dans le Grès Vosgien, à des profondeurs variant de 5 à $80 \mathrm{~m}$, consécutivement à une dégradation du recouvrement et à l'ennoyage des galeries, puis de quantifier les effets de surface que d'éventuelles instabilités souterraines pouvaient induire.

Une première analyse a permis de faire la distinction entre deux types d'effet susceptibles de se manifester : les effondrements localisés, qui se traduisent par l'apparition brutale d'un fontis à la surface, et les affaissements, qui sont la conséquence d'une déformation plus progressive des terrains de recouvrement. Les mécanismes conduisant à l'apparition de ces phénomènes et les paramètres les conditionnant ont été explicités.

Le recours à la modélisation numérique capable de simuler le comportement hydromécanique d'un milieu rocheux poreux ou fracturé (codes FLAC et UDEC) nous a permis de répondre plus précisément à cet objectif.

Nous avons distingué trois configurations géologiques qui rendent compte des disparités régionales en ce qui concerne la stratification et la fracturation du grès.

La dégradation du recouvrement a été simulée en supposant une diminution très importante des caractéristiques de raideur et de résistance du grès. La simulation de la remontée des eaux vers la surface a été réalisée de façon progressive, en tenant compte de la phase transitoire pendant laquelle la galerie n'est pas totalement noyée: On a pu établir que :

- les effets de surface sont négligeables lorsque les galeries sont creusées dans des bancs de grès épais et peu fracturés (configuration 1), même lorsqu'elles sont situées à très faible profondeur :

- dans des grès très stratifiés (configuration 2), les effets sont un peu plus sensibles tout en restant de faible amplitude. Les affaissements et les déformations horizontales calculés sont d'ailleurs largement surestimés par les hypothèses exagérément pessimistes d'une altération extrême du grès, et d'une raideur très faible des joints de stratification ;

- lorsque les galeries traversent des zones de faille (configuration 3), les risques d'instabilité ou d'apparition de fontis à la surface peuvent être, a priori, plus importants du fait d'une fracturation supplèmentaire du grès. Les simulations réalisées ont pu montrer que, si cette fracturation n'est pas trop importante, la hauteur de la cloche d'éboulement qui se forme au toit de la galerie reste faible, ainsi que les effets ressentis a la surface. Si la fracturation du grès est plus intense, on prévoit l'apparition de fontis uniquement si les galeries sont très proches de la surface et si les caractéristiques des fractures sont très faibles. Dès que la profondeur dépasse $8 \mathrm{~m}$, la montée de la cloche d'éboulement est stoppée avant d'atteindre la surface par effet de foisonnement.

Les effets liès à la mise en eau du recouvrement peuvent induire un endommagement du grès à proximité de la galerie pour des profondeurs supérieures à $30 \mathrm{~m}$ si le grès est massif (configuration 1), supérieures à $20 \mathrm{~m}$ si le grès est constitué de bancs d'épaisseur métrique (configuration 2). Dans ces deux configurations, pratiquement aucun effet lié à cet endommagement n'est perçu à la surface. Dans les zones de faille (configuration 3), les perturbations supplémentaires liées à la présence de l'eau sont négligeables devant celles déjà constatées préalablement.

\section{5}

\section{Traitement des galeries : application à l'ancienne galerie de Falck}

\section{1}

\section{Principes de mise en sécurité des galeries}

Des résultats des analyses précédentes, il ressort clairement que la stabilité sur le long terme des anciennes galeries minières creusées dans la formation des grès vosgiens est fortement conditionnée par la structure (stratification et densité de fracturation) et l'état d'altération du massif gréseux. D'autre part, toutes choses égales par ailleurs, plus une galerie est profonde moins les effets d'une éventuelle instabilité de celle-ci seront de forte amplitude voire même ressentis en surface. Ainsi, tant que nous avons affaire à un massif gréseux cohérent et peu altéré, peu à moyennement stratifié et/ou fracturé, on considère qu'au-delà d'une vingtaine de mètres de profondeur, un éboulement affectant une galerie n'atteindra pas la surface par simple effet voûte ou par auto-comblement de la galerie et de la cloche d'éboulement par foisonnement des terrains éboulés. Il n'est donc pas préconisé de mesures compensatoires particulières pour garantir la stabilité des galeries creusées dans ce type de terrain. Seuls leurs orifices débouchant au jour doivent être traités jusqu'à une profondeur n'excédant pas la dizaine de mètres (Wojtkowiak et Didier, 1999).

Toujours en s'appuyant sur les résultats des modélisations numériques, les Houillères du bassin de Lorraine portent, par contre, une attention particulière aux galeries peu profondes, situées à moins d'une vingtaine de mètres de profondeur et creusées dans des grès qui ne présentent pas, sur le plan géologique et géotechnique, de garanties suffisantes pour conclure à leur stabilité sur le long terme. Dans ces conditions, des mesures compensatoires doivent être prises pour la mise en sécurité des galeries concernées et des terrains de surface. C'est plus particulièrement le cas des galeries peu profondes creusées dans des grès très fracturés et/ou très altérés. Nous avons vu précédemment que, dans certains secteurs du bassin houiller lorrain, les conditions de dépôt de ces faciès gréseux et/ou leur altération ultérieure ont été telles que ces formations sont pratiquement dépourvues de cohésion et assimilables, en terme de comportement mécanique, à des matériaux sableux. Il va de soi que, dans de telles conditions défavorables à leur stabilité, les galeries qui traversent ce type de faciès doivent faire l'objet de travaux de traitement qui font généralement appel aux techniques de comblement partiel ou intégral.

A titre d'exemple de galerie située dans un tel contexte, nous présentons les reconnaissances préalables puis les travaux réalisés pour le traitement et la mise en sécurité de l'ancienne galerie d'exhaure de Falck. 


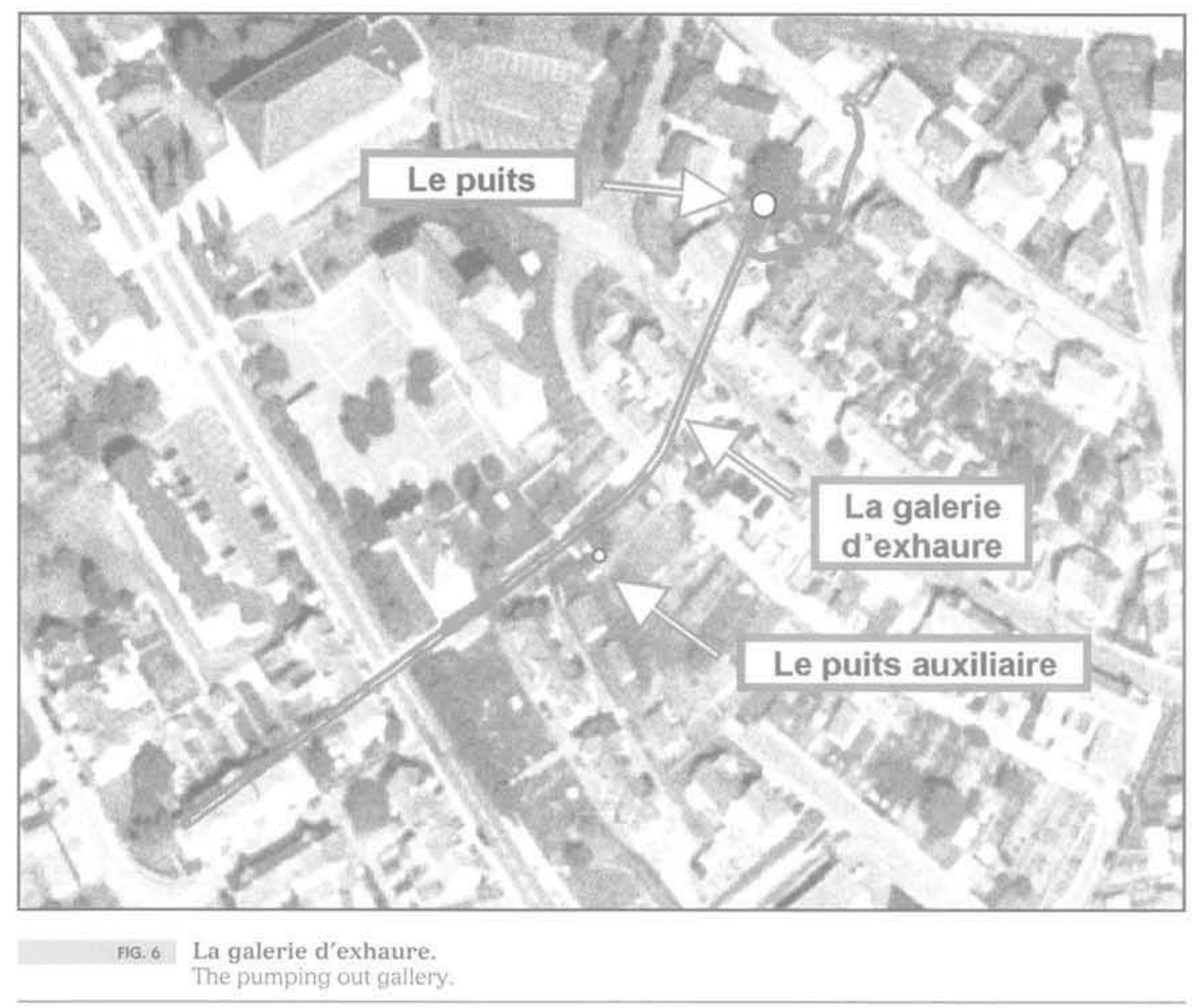

\section{2}

\section{Historique de la galerie de Falck}

En 1856, la Socièté de Recherche de Falck entreprend des sondages et découvre des terrains houillers productifs sur le ban de la commune de Falck. La Compagnie Houillère de Falck est constituée en 1858 pour exploiter le gisement. L'État lui accorde la concession de Falck par décret impérial du 2 juillet 1859. La compagnie n'attend pas l'obtention de la concession pour entreprendre, dès sa création en 1858, le fonçage d'un puits de $4,20 \mathrm{~m}$ de diamètre dans la formation des qrès vosgiens. A la profondeur de $19,5 \mathrm{~m}$, une galerie d'épuisement des eaux est creusée sur une longueur de $212 \mathrm{~m}$. Elle débouche sur un petit vallon. La galerie a une hauteur de $2 \mathrm{~m}$ et une largeur de $1,8 \mathrm{~m}$. Une rigole carrée de 0,6 m de côté est creusée au parement nord. La galerie a été creusée simultanément à partir du puits principal, où une recette de $6 \mathrm{~m}$ de haut et $4 \mathrm{~m}$ de profondeur a été excavée, et d'un puits auxiliaire. En 1860, les actionnaires décident d'arrêter le fonçage à la profondeur de $73 \mathrm{~m}$, à cause de venues d'eau importantes, occasionnant des frais jugés intolérables. Les bâtiments en tête de puits, devenus dangereux, sont démolis en 1896. Le carreau et le puits principal sont remblayés en 1931.

L'ouvrage se trouve à présent en site totalement urbanisé, au coeur de la commune de Falck (Fig. 6), La galerie est redécouverte accidentellement en 1982, des désordres étant survenus dans un jardin privé au droit du puits auxiliaire.

Le service juridique des HBL a examiné la question de la responsabilité de la houillère vis-à-vis de cet ouvrage. La concession de Falck est devenue, par fusion avec quatre autres petites concessions, la concession de Dalem par arrêté du commissaire de la République de Strasbourg daté du 31 août 1920. Cette concession, propriété de l'État, a été transférée aux HBL par décret $n^{\circ}$ 47-621 du 5 avril 1947. Dans ces conditions, les HBL sont responsables de cet ouvrage et procèdent alors au levé de l'ouvrage à partir du puits auxiliaire en 1982.

En 2000, des propriétaires s'inquiètent de fissures observées sur leurs maisons. Bien qu'a priori sans rapport avec l'ouvrage, les HBL décident néanmoins de traiter l'ouvrage pour mettre le site définitivement en sécurité.

\section{3}

\section{Traitement de l'ouvrage}

Les HBL s'appuient pour définir les principes de traitement de tous les ouvrages creusés dans les grès vosgiens, sur les résultats de l'étude réalisée en 1997 par I'TNERIS pour évaluer les effets de surface dus aux galeries creusées à très faible profondeur. Nous avons vu que, dans le contexte de cette étude, la stabilité d'une galerie de faible section est assurée, selon les résultats de calcul, dès lors que le recouvrement dépasse huit mètres d'épaisseur de grès relativement résistants.

Vu la sensibilité du site, totalement urbanisé, il a été décidé de reconnaitre plus précisément l'ouvrage, en vue de définir les principes de son traitement et garantir ainsi la sécurité des personnes et des biens dans son environnement immédiat. 


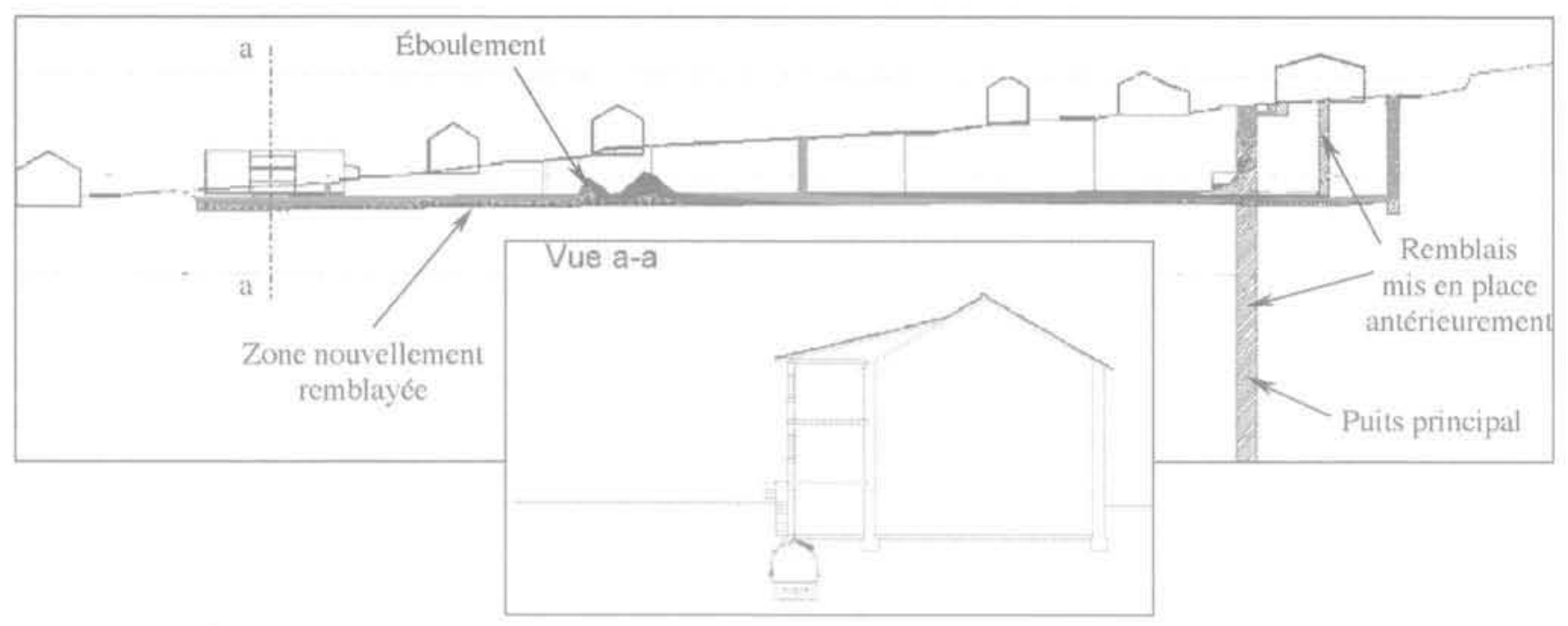

FIG.7 Profil de l'ouvrage.

Profile of the work.

II apparait dès le premier sondage de reconnaissance réalisé en décembre 2001 que la position de la galerie repérée sur les levés de 1982 est incorrecte. Un levé au GPS montre en effet un écart de $3,40 \mathrm{~m}$ au niveau du puits auxiliaire. Cette correction prise en compte, les sondages reprennent mais ne localisent toujours pas l'ouvrage, dont l'axe est également mal levé.

L'équipe de sauveteurs des HBL est alors mobilisée (la teneur en oxygène dans la galerie étant à la limite réglementaire de $19 \%$ ) et intervient pour rendre la galerie accessible. Cette équipe opère le déblaiement à la main du sable qui encombrait la galerie de manière à laisser écouler l'eau qui ennoyait partiellement l'ouvrage. Un nouveau levé a pu alors être effectué par les geomètres de l'UE La Houve avec l'assistance des sauveteurs. Il est toutefois impossible de lever avec précision la position de l'extrémité orientale de la galerie, restée partiellement noyée et de trop faible hauteur libre pour y travailler.

La figure 7 illustre le profil de l'ouvrage établi à partir des résultats des reconnaissances par sondages et des levés effectués. On notera que sa profondeur varie de $3 \mathrm{~m}$ seulement à $17 \mathrm{~m}$. L'extrémité orientale finit sur un deuxième puits auxiliaire qui n'était pas mentionné dans les archives. Ce puits a été retrouvé au jour par sondages effectués $\alpha$ à tâtons $)$.

Compte tenu de l'ensemble de ces données, de la vulnérabilité de la surface et des risques de mouvements de terrain liés à la présence de cette galerie, localement très peu profonde et creusée dans une formation de grès très altérée et par conséquent peu cohérente, il a été décidé de remblayer intégralement l'ouvrage sur toute sa lonqueur. Les travaux ont démarré en décembre 2001 et ont consisté en des injections d'un coulis cendres-ciment. Ce coulis a été injecté dans les cavités par des trous de sonde tubés. Au total, près de $1100 \mathrm{~m}^{3}$ de coulis ont été utilisés et les travaux se sont achevés fin avril 2002. Notons qu'au cours de ces travaux, ont été retrouvés : le pied d'un ouvrage circulaire en briques, probablement une ancienne cheminée : une petite galerie auxiliaire d'usage inconnu, non mentionnée sur les plans de mine ainsi qu'un vide au-dessus de la voûte de la galerie, peut-être dû à l'altération 110 météorique et l'érosion par les eaux d'infiltration des terrains encaissants (ce vide a été exploré par caméra). Tous ces vides et ouvrages annexes ont également été remblayés. Enfin, on retiendra que la position réelle du puits est en fait à une distance d'une quarantaine de mètres du point figurant dans les archives disponibles.

\section{Conclusion}

L'objectif de l'étude était :

- d'analyser les conditions de stabilité des galeries creusées dans la formation des grès vosgiens à des profondeurs variant de 5 à $80 \mathrm{~m}$, consécutivement à une dégradation du recouvrement et à l'ennoyage des galeries, puis de quantifier les effets de surface que d'éventuelles instabilités souterraines pouvaient induire :

- de préconiser, le cas échéant, un traitement adapté de ces galeries dans les zones à risque.

Dans le cas le plus défavorable étudié, correspondant à un massif gréseux stratifiè, movennement fracturé et altéré, les analyses réalisées prédisent l'apparition de fontis en surface uniquement si les galeries sont très peu profondes et si les caractéristiques géomécaniques des grès et des discontinuités naturelles qui les affectent sont très faibles. Dans le cas contraire, dès que la profondeur de la galerie dépasse 8 mètres, la remontée de la cloche d'éboulement est bloquée avant d'atteindre la surface par l'effet de foisonnement des terrains éboulés. Il n'est donc pas préconisé de mesures compensatoires particulières pour garantir la stabilité des galeries creusées dans ce type de formation gréseuse. Seuls leurs orifices débouchant au jour doivent être traités jusqu'à une profondeur n'excédant pas la dizaine de mètres.

Toujours en s'appuyant sur les résultats des modélisations numériques, les Houillères du bassin de Lorraine portent, par contre, une attention particulière aux galeries peu profondes, situées à moins d'une vingtaine de mètres de profondeur et creusées dans des grès très fracturés et/ou très altérés qui, de ce fait, ne présentent pas, sur le plan géologique et géotechnique, de garanties suffisantes pour conclure à leur 
stabilité sur le long terme. Dans ces conditions, des mesures compensatoires doivent être prises pour la mise en sécurité de ces galeries et, par conséquent, des terrains de surface. C'est plus particulièrement le cas des galeries peu profondes creusées dans des faciès dont les conditions de dépôt et/ou leur altération ultérieure ont été telles que ces formations sont pratique- ment dépourvues de cohésion et assimilables, en terme de comportement mécanique, à des matériaux sableux. Il va de soi que, dans de telles conditions défavorables à leur stabilité, les galeries qui traversent ce type de faciès doivent faire l'objet de travaux de traitement qui font généralement appel aux techniques de comblement partiel ou intégral.

\section{$\overline{\text { Bibliographie }}$}

Bertrand L., Bonvallet J., Both I., Feuga B. et Wojtkowiak F-Etude de linfluence d'un trafic routier de fort tonnage sur la stabilité de carrières souterraines abandonnées en Gironde. Détermination du confortement à mettre en œuvre. Deuxièmes journèes nationales géotechniques, Nantes. Industrie minerale, juin, 1982, p. 1-14.

BRGM - Carte géologique de la France au $1 / 50000$ - région LEMBACH. BRGM, Service géologique national, 1976.

Homand F. - Comportement mécanique des roches en fonction de la température. Sciences de la Terre, Mémoire $n^{\circ} 46$. juin 1985.

ITASCA Consulting Group - User's Manual UDEC (Version 2.0) \& FLAC (Version 3.3), 1993.
Mahmoud Y.S.E. - Aralyse de l'influence des différents paramètres d'exploitation et facteurs naturels sur le comportement des terrains dans lexploitation par longues. tailles. Thèse de docteur ingênieur. Laboratoire de mécanique des terrains, École des mines de Nancy, INPL. 1984.

Maury V.- Effondrements spontanés Synthèse d'observations et possibilite de mécanismes initiateurs par mise en charge hydraulique. Revue de lindustric minérale, octobre 1979 .

Piguet J.P. - Evaluation des effers de surface dus aux voies et galeries minières anciennes du houiller. Rapport INERIS non public, Réf. SSE-JPP/DK-97. 26EE54/RN01, avril 1997.

Piguet J.P. Wojtkowiak F. - Affaissements et déformations au-dessus des exploitations minières : mécanismes et évolution dans le temps. Les Techniques de l'industrie minè. rale. $n^{\circ} 10$, fuin 2001; p. 10-22.

Pineau J.L, - Influence de l'eau sur les propriétés physicomécaniques d'un grès DEA. Génie géologique et minier, INPL. 1976.

Thoraval A. - Evaluation des effets de surface dus aux galeries creusées á très faible profondeur dans les grès vosgiens. Rapport RNERIS non public. Réf. SSEATh/Dmi 97-26 EF 43/FN01, avril 1997.

Vachat J.C. - Les désordres survenant dans les carrières de la région parisienne. Mémoire diplòme ingénieur CNAM, Paris, 1982

Woitkowiak F., Didier C. (1999). Principes de traitement d'anciens ouvrages miniers débouchant en surface. Comptes rendus du 9. conarés international SIMR. Paris, 1999, p. 25-30. 